\title{
THE STATE OF THE SECOND PART OF HILBERT'S FIFTH PROBLEM
}

\author{
J. ACZÉL
}

1. Introduction. In his celebrated address to the 1900 International Congress of Mathematicians, about unsolved problems, Hilbert (1902) stated the second part of his fifth problem as follows.

"Moreover, we are thus led to the wide and interesting field of functional equations which have been heretofore investigated usually only under the assumption of the differentiability of the functions involved. In particular the functional equations treated by Abel (Oeuvres, vol. 1, pp. $1,61,389$ ) with so much ingenuity... and other equations occurring in the literature of mathematics, do not directly involve anything which necessitates the requirement of the differentiability of the accompanying functions... In all these cases, then, the problem arises: In how far are the assertions which we can make in the case of differentiable functions true under proper modifications without this assumption?" (Hilbert's emphasis).

As in some others in that series of Hilbert problems, the question is not uniquely defined. If, however, we concentrate on Abel's results, as Hilbert did, we can make a definite judgement on how far we got in the direction recommended by Hilbert and that is what I plan to do here.

Abel had four publications and three manuscripts containing functional equations.

We try to look at most, if not all functional equations on which Abel worked and got results. In $\S 2$ we deal with two problems for which Abel did not suppose differentiability but where recent results still succeeded in reducing the regularity suppositions.

$\S \S 3$ and 4 are the main part of this survey: it reports on results where the Hilbert program has been carried through: the differentiability conditions used by Abel have been successfully replaced, some quite recently, by (much) weaker conditions.

Finally, in the second part of $\S 4$, I speak about a system of two functional equations with which Abel dealt by way of differentation, and which pair has since been reduced to just one of the two equations but where differentiability has not been eliminated yet, so that is still missing to the complete solution of Hilbert's problem.

Received by the editors November 2, 1988.

1980 Mathematics Subject Classification (1985 Revision). Primary 39B30; Secondary 01A55, 01A60, 01A65, 39B10, 39B20, 39B40, 39B70. 
2. The complex exponential equation and Abel's equation.

1. The important paper Abel (1826B) justifies 'Newton's binomial series' in its full generality (avoiding also an error in Cauchy (1821) who neglected to prove uniform convergence) solving the complex exponential functional equation

$$
f(z+w)=f(z) f(w) \quad(z, w \in \mathbf{C})
$$

for continuous complex-valued functions of a complex variable. No differentiability has been assumed there. However, using an idea of Kac (1937), the continuity supposition can be weakened to measurability in the following way.

It is well known (and obvious) that a solution of (1) is either everywhere or nowhere 0 . Let us exclude the trivial solution

$$
f(z)=0 \quad \text { for all } z \in \mathbf{C}
$$

for the time being. Starting with a slightly simpler problem, we restrict (1) first to real variables:

$$
f(x+t)=f(x) f(t) \quad(x, t \in \mathbf{R})
$$

with $f$ still complex-valued. This can, of course, be written as the pair of equations $(r(x)=|f(x)|>0, g(x)=f(x) /|f(x)|)$,

$$
\begin{array}{ll}
r(x+t)=r(x) r(t) & \left(x, t \in \mathbf{R} ; r: \mathbf{R} \rightarrow \mathbf{R}_{+}\right), \\
g(x+t)=g(x) g(t) & \left(x, t \in \mathbf{R} ; g: \mathbf{R} \rightarrow e^{i \mathbf{R}}\right)
\end{array}
$$

with

$$
|g(x)|=1 \quad(x \in \mathbf{R}) .
$$

The function $f$ and thus also $g$ is now supposed to be just measurable on a proper real interval. Let $[a, b]$ be such an interval for (5). Since, by (6), $g$ is also bounded, it is Lebesgue integrable. So let us integrate (5) with respect to $t$ from $a$ to $c$, where $c$ is chosen so that $C=\int_{a}^{c} g(t) d t \neq 0$. (Such a $c$ exists for otherwise $\int_{a}^{x} g(t) d t \equiv 0$ so $g(x)=0$ a.e. on $[a, b]$, contrary to (6).) With a change of variables $(s=x+t)$ we get

$$
g(x)=\frac{1}{C} \int_{x+a}^{x+c} g(s) d s .
$$

Since $g$ is integrable, the right-hand side is a continuous function of $x$ and so is the left, that is, $g$. But, if $g$ is continuous, then the right-hand side is differentiable and so is the left. Since $g$ is now differentiable, we may differentiate (5) with respect to $t$ and then substituting $t=0$ we get the differential equation

$$
g^{\prime}(x)=B g(x) \quad(x \in \mathbf{R})
$$

with the general complex valued solution $(A, B$ complex constants)

$$
g(x)=A e^{B x},
$$

which satisfies (5) (and is not identically zero) iff $A=1$ while (6) is satisfied iff $B=i \beta$ where $\beta$ is a real constant. 
So the general measurable solution of (5) and (6) is given by

$$
g(x)=e^{i \beta x} \quad(x \in \mathbf{R} ; \beta \text { a real constant }) .
$$

As to (4), its measurable nonzero solution is well known to be

$$
r(x)=e^{\alpha x} \quad(x \in \mathbf{R} ; \alpha \text { a real constant }) .
$$

[Kac (1937) proved this by reduction to the above argument for $\tilde{g}(x)=$ $e^{i \log r(x)}$. Actually, this yields only $e^{i \log r(x)}=e^{i \alpha x}$, that is,

$$
\log r(x)=\alpha x+2 k(x) \pi,
$$

where $k(x) \in \mathbf{Z}$. But $x \mapsto \alpha x$ and also $x \mapsto \log r(x)$ are linear for rational coefficients, so $k$ has to be linear too and is thus 0 , so that $r(x)=x^{\alpha}$.] From (7) and (8)

$$
f(x)=e^{(\alpha+i \beta) x}=e^{D x},
$$

( $D$ an arbitrary complex constant) is the general not identically 0 measurable solution of (3).

As to (1), it implies

$$
f(z)=f(x+y i)=f(x) f(y i),
$$

where both factors, this time as functions of the real variables $x$ or $y$, satisfy (3), so all measurable solutions of (1) are given by

$$
f(z)=f(x+y i)=e^{D x+E y}=e^{\delta z+\varepsilon z} \quad(z \in \mathbf{C}),
$$

$(D, E, \delta, \varepsilon$ arbitary complex constants) and by (2).

Note that here we derived from measurability continuity and, eventually, differentiability (on R). We will come back to this method (cf. Aczél (1961), Járai (1986)) in 3.2.

2. Abel's (1824) manuscript deals with the 'Abel equation'

$$
f[\phi(x)]=f(x)+1 .
$$

This has by now an immense literature. We refer the reader to Kuczma (1968) and Kuczma, Choczewski and Ger (1989). We quote here just a result of Zdun (1977).

Let $\phi$ be continuous, convex and strictly increasing on $[0, a], 0<\phi(x)<$ $x$ for $x \in] 0, a]$ and let $\phi^{n}$ be the $n$-th iterate of $\phi$. Then equation (9) has an integrable solution $f$ if, and only if, $\sum_{n=1}^{\infty} \phi^{n}\left(x_{0}\right)$ converges for some $\left.\left.x_{0} \in\right] 0, a\right]$. This solution depends on an arbitrary integrable function $f_{0}$ on ] $\phi(a), a]$ (meaning that for every such $f_{0}$ there is exactly one solution $f$ of (9), integrable on $] 0, a$, whose restriction to $] \phi(a), a]$ is $f_{0}$ ).

\section{Elimination of the differentiability conditions in some important works} of Abel.

1. Another important 1826 paper of Abel (1826A) solves the system of functional equations

$$
\begin{aligned}
F[x, F(y, z)] & =F[z, F(x, y)]=F[y, F(z, x)]=F[x, F(z, y)] \\
& =F[z, F(y, x)]=F[y, F(x, z)]
\end{aligned}
$$

by reducing it to partial differential equations. So here differentiability was indeed supposed and used in an essential way. Nowadays this system 
is considered as a combination of associativity and commutativity. The associativity equation

$$
F[x, F(y, z)]=F[F(x, y), z]
$$

has been completely solved for $x, y, z \in I$ (a real interval; there are generalizations for continuous and/or ordered structures, cf. e.g. Fuchs (1963)) under continuity and cancellativity conditions but without supposing differentiability or even commutativity (Aczél (1949), (1966), (1987)): commutativity is a consequence. The proof used to be rather lengthy but recently Páles and Craigen (1989) found a shorter proof. (We mention here that a book on associativity-mostly with other additional conditions-by C. Alsina, M. J. Frank and B. Schweizer is in preparation.)

We give here the theorem and a sketch of the Páles-Craigen (1989) proof.

Let $I \subset \mathbf{R}$ be a proper interval. The function $F: I^{2} \rightarrow I$ is continuous, associative (10) and cancellative $\left(\left(\exists y \in I: F\left(x_{1}, y\right)=F\left(x_{2}, y\right)\right) \Rightarrow x_{1}=\right.$ $\left.x_{2} ;\left(\exists x \in I: F\left(x, y_{1}\right)=F\left(x, y_{2}\right)\right) \Rightarrow y_{1}=y_{2}\right)$ if, and only if, there exists a continuous strictly monotonic $f: I \rightarrow \mathbf{R}$ such that

$$
F(x, y)=f^{-1}(f(x)+f(y)) \quad(x, y \in I) .
$$

For the sake of brevity we write $x \circ y:=F(x, y)$ and define $x^{n}$ by $x^{1}=x, x^{n+1}=x^{n} \circ x(n=1,2, \ldots)$.

The proof proceeds through the following steps.

(i) $F$ is (strictly) increasing in each variable. Since $I$ is a proper interval there exists a $c \in I$ for which $c \circ c \neq c$. Let us take, say, $c<c \circ c$.

(ii) $\left\{x \circ c^{n}\right\}$ is increasing and $\lim _{n \rightarrow \infty}\left(x \circ c^{n}\right)=\sup I \notin I$. (As a consequence, $I$ is open from above; if we had assumed the existence of a $d>d \circ d$ then $I$ would have turned out to be open from below.) This follows from (i).

(iii) The inequalities $c^{m}>x \circ c^{n}$ and $c^{m}>c^{n} \circ x$ are equivalent for each $x \in I, m, n \in \mathbf{N}$. This too follows from (i).

(iv) If, for some $x, y \in I, m, n, k, l \in \mathbf{N}, c^{m}>x \circ c^{n}$ and $c^{k}>y \circ c^{l}$ then $c^{m+k}>x \circ y \circ c^{n+l}$. Furthermore the inequalities $c^{m}>x \circ c^{n}$ and $c^{m k}>x^{k} \circ c^{n k}$ are equivalent. (These follow from (iii).)

(v) For all $x, y \in I, n \in \mathbf{N}$,

$$
\min \left(x^{n} \circ y^{n}, y^{n} \circ x^{n}\right) \leq(x \circ y)^{n} \leq \max \left(x^{n} \circ y^{n}, y^{n} \circ x^{n}\right) \text {. }
$$

We prove this in the case $x \circ y<y \circ x$ (the proof is similar for $x \circ y>y \circ x$; there is nothing to prove for $x \circ y=y \circ x)$ :

$$
\begin{aligned}
(x \circ y)^{n} & =x \circ y \circ x \circ y \circ \cdots \circ x \circ y<y \circ x \circ y \circ x \circ \cdots \\
& <y \circ y \circ x \circ x \circ y \circ \cdots<\cdots<y^{n} \circ x^{n} \\
(x \circ y)^{n} & =x \circ y \circ x \circ y \circ x \circ \cdots>x \circ x \circ y \circ x \circ y \circ \cdots \\
& >\cdots>x^{n} \circ y^{n} .
\end{aligned}
$$

We start now to construct $f$ for (11). For $x \in I$ we define

$$
\Phi_{x}=\left\{r=\frac{m-n}{k} \mid m, n, k \in \mathbf{N}, c^{m}>x^{k} \circ c^{n}\right\}
$$


(by (iv) this definition does not change if the same $r$ is represented by different $m, n, k$ ). By (ii), $\lim c^{m}>x \circ c$, so $\exists m^{\prime} \in \mathbf{N}$ in $\Phi_{x}$, which thus is not empty. If $r \in \Phi_{x}$ then every rational $r^{\prime}>r$ is also in $\Phi_{x}$. So $\Phi_{x}$ consists of the rational numbers of an interval unbounded from above. By (ii) $\exists n: c<x \circ c^{n}$, so $(1-n) / 1$ is a lower bound of $\Phi_{x}$. Define

$$
f(x)=\inf \Phi_{x} .
$$

(vi) $f(x \circ y)=f(x)+f(y)(x, y \in I)$. (This follows from (iv) and (v).) (vii) $f$ is continuous and strictly monotonic.

Now (11) follows from (vi) and (vii) and the proof is complete.

As relevant to Abel's (1826A) original system of equations we mention also the functional equation of commuting systems of transformations $F: S \times Z \rightarrow Z:$

$$
F[x, F(y, z)]=F[y, F(x, z)] \quad(x, y \in S, z \in Z)
$$

which has been solved in quite general situations, for instance where $S$ and $Z$ are compact, $F$ is continuous and a 'weak transitivity' holds, that is, there exists a $c \in Z$ such that for all $z \in Z$ there is a $y$ with $F(y, c)=z$ (see Aczél-Wallace (1967)). The result is that it is necessary and sufficient that $S$ be a commutative monoid, with $c$ as unit, under an operation $\circ$ and that there exist a continuous surjection $f: S \rightarrow Z$ (actually, $f(x)=F(x, c)$ ), such that

$$
F(x, z)=f(x) \circ z .
$$

2. Abel (1823) presented a general method of solving functional equations of quite general forms by reduction to differential equations. Of course he tacitly assumed that the unknown functions are several times differentiable. (Some of the steps in Abel (1823) look outlandish but can be justifed, see Kiesewetter (1957).)

However, in the spirit of Hilbert's (1902) program, one may reduce Abel's regularity conditions by showing that weak regularity conditions (say measurability) and the fact that the unknown function(s) satisfy certain functional equations imply their differentiability of the required order. Kac's (1937)s above mentioned method does this for Cauchy's equation. A similar method (but supposing continuity) has been applied already by Andrade (1900) to d'Alembert's (Poisson's) equation

$$
f(x+y)+f(x-y)=2 f(x) f(y) .
$$

(An equation equivalent to this was solved by Abel (1823), using his method of derivations.) This method can be generalized to fairly broad classes of equations (see e.g. Aczél (1961), (1966), Aczél and Chung (1982), Aczél and Dhombres (1989)).

Recently Járai (1986) has obtained nice results encompassing wide classes of equations. Here are some typical classes ( $f$ is the unknown functions),

$$
\begin{gathered}
f(x)=\sum_{j=1}^{n} h_{j}\left(x, y, f\left[g_{j}(x, y)\right]\right) \\
f(x)=h\left(x, y, f(y), f\left[g_{1}(x, y)\right], \ldots, f\left[g_{n}(x, y)\right]\right) .
\end{gathered}
$$


He goes through the following steps: (I) measurability (or Baire property) implies continuity; (II) continuity implies the local Lipschitz condition; (III) from that, $C^{1}$ (continuous differentiability) follows and (IV) $C^{1} \Rightarrow$ $C^{\infty}$ (differentiability of any order). All these are done under rather weak conditions, except step (II) which works for Járai (1986) only for (12), the more special of the above equations.

Abel (1823) shows on several examples how his method works. In addition to d'Alembert's equation, mentioned above, he deals in this way with the 'equation of the logarithm', equivalent to (4) and 'solves' also the 'arc tan equation'

$$
f(x)+f(y)=f\left(\frac{x+y}{1-x y}\right) \quad(x, y \in \mathbf{R}) .
$$

However, this equation has no continuous solutions except $f(x) \equiv 0$, as Kiesewetter (1965) showed (there exist local solutions and global but multivalued solutions). Losonczi (1985) gave the general solutions (they are not all 0 but Kiesewetter's result is an immediate consequence of Losonczi's). The two remaining examples in Abel (1823), which do not contain derivatives in the first place, are

$$
\phi(x+y)=\phi(x) f(y)+f(x) \phi(y)
$$

and

$$
\psi(x+y)=g(x y)+h(x-y) .
$$

By now the general solutions of each of these can be determined without any regularity condition. The first has been solved on arbitrary semigroups (not even commutativity is supposed) by Chung, Kannappan and $\mathrm{Ng}$ (1985). The second can be written, with the notations $t=(x-y)^{2}$, $s=4 x y, \phi(s)=g(s / 4), f(t)=h(\sqrt{t})$ (for $t>0), k(z)=\psi(\sqrt{z})$ (for $z>0)$ as

$$
k(s+t)=\phi(s)+f(t)
$$

(the so called Pexider equation) on the domain $\{(s, t) \mid s+t>0, t>0\}$. But the Pexider equation (14) can be extended uniquely from any (open) region to all of $\mathbf{R}^{2}$ (see e.g. Radó and Baker (1987)) and its general solution is given by

$$
\phi(s)=a(s)+\alpha, \quad f(t)=a(t)+\beta, \quad k(z)=a(z)+\alpha+\beta,
$$

so that the general solution of $(13)$ is given by

$$
\begin{gathered}
g(u)=\phi(4 u)=4 a(u)+\alpha, \quad h(v)=f\left(v^{2}\right)=a\left(v^{2}\right)+\beta, \\
\psi(w)=k\left(w^{2}\right)=a\left(w^{2}\right)+\alpha+\beta,
\end{gathered}
$$

where $\alpha, \beta$ are arbitrary constants and $a$ is an arbitrary additive function. If one of $g, h, \psi$ is even mildly regular (say measurable), then

$$
a(t)=\gamma t
$$

which gives exactly Abel's (1823) solutions. (Cf. also Lajkó 1987.) 
3. A manuscript of Abel (1826C) contains the system of functional equations

$$
\begin{aligned}
& \psi\left(\frac{x}{1-x} \frac{y}{1-y}\right)= \psi\left(\frac{y}{1-x}\right)+\psi\left(\frac{x}{1-y}\right) \\
&-\psi(x)-\psi(y)-\log (1-x) \log (1-y), \\
& \psi(x)+\psi(1-x)=C-\log x \log (1-x) .
\end{aligned}
$$

(If 1 is in the domain then the second equation follows from the first with $y=1-x, C=\psi(1)$.)

Kiesewetter (1961) reduces them to the equation

$$
F(u)+F(v)+F\left(\frac{1-u}{1-u v}\right)+F(1-u v)+F\left(\frac{1-v}{1-u v}\right)=0
$$

If $u=0, v=0$ are also permitted, Daróczy and Kiesewetter (1973) find the general (Lebesgue) integrable solution of (15). They first apply the Andrade(1900)-Kac(1937) method (cf. $\S \S 2.1,3.2$ above). They integrate with respect to $v$ from 0 to 1 . We will do so from $\delta$ to $1-\delta$ ( $\delta$ small):

$$
\begin{gathered}
(1-2 \delta) F(u)=-\int_{\delta}^{1-\delta} F(t) d t-\frac{1-u}{u} \int_{(1-u) /(1-\delta u)}^{(1-u) /(1-(1-\delta) u)} t^{-2} F(t) d t \\
-\frac{1}{u} \int_{1-(1-\delta) u}^{1-\delta u} F(t) d t-(1-u) \int_{\delta /(1-(1-\delta) u)}^{(1-\delta) /(1-\delta u)}(1-u t)^{-2} F(t) d t
\end{gathered}
$$

Since the right-hand side is continuous in $u$ on $] 0,1$, so is the left, that is, $F(u)$. But then the right-hand side is differentiable and so is $F$. They stop here but, with differentiable $F$, the right-hand side, and with it $F(u)$, is twice, then three, four... times differentiable on $(0,1)$ (we will need the existence of $\left.F^{\prime \prime \prime}\right)$. They now differentiate (15) with respect to $u$ and get, for

$$
f(t)=t(1-t) F^{\prime}(t)
$$

the equation

$f(u)-f\left(\frac{1-u}{1-u v}\right)-\frac{1-u}{1-u v} f(1-u v)+u f\left(\frac{1-v}{1-u v}\right)=0 \quad(u, v \in] 0,1[)$.

With the substitution $x=1-u, y=1-(1-u) /(1-u v)$ this becomes

$$
\begin{array}{r}
f(1-x)+(1-x) f\left(\frac{y}{1-x}\right)=f(1-y)+(1-y) f\left(\frac{x}{1-y}\right) \\
(x, y, x+y \in] 0,1[) .
\end{array}
$$

They need (15) for $v=0$ in order to prove $f(1-t)=f(t)$ with which (17) goes over into the so called fundamental equation of information and use a deeper result of Lee (1964) on the general measurable solution. But we know that $F$ and so, by (16), $f$ is as often differentiable as needed. Differentiating (17) with respect to $x$, then $y$, we get

$$
t(1-t) f^{\prime \prime}(t)=c \quad(c \text { constant })
$$


and thus

$$
f(t)=c(t \log t+(1-t) \log (1-t))+a t+b(t \in] 0,1[),
$$

which satisfies (17) iff $b=-2 a$. By (16),

$$
F(t)=c \int_{\alpha}^{t}\left(\frac{\log x}{1-x}+\frac{\log (1-x)}{x}\right) d x-a \log \frac{t^{2}}{1-t}
$$

linking $F$ to the dilogarithm. [Cf. Kiesewetter (1961). - Daróczy and Kiesewetter (1973) found the first term as general solution of (15) for $u, v \in[0,1]$ (on larger domains a functional equation may have fewer solutions), but noted that for $u, v \in] 0,1$ [ the second term satisfies it too. Cf. also Kannappan $(1977,1978)$. Járai's (1986) methods make it possible to reduce the integrability assumption to measurability.

4. Recently solved and partially solved problems.

1. In the paper Abel (1827), the functional equation

$$
\phi(x)+\phi(y)=\psi[x f(y)+y f(x)]
$$

is solved by repeated differentiation. This is one of the places where Abel notes the remarkable fact that one functional equation determines several unknown functions. The conclusion he draws from this fact is equally remarkable:

"Thus, it is generally possible to find all the functions by means of a single equation. It follows that such an equation can exist only very seldom. Indeed, since the form of an arbitrary function appearing in the given conditional equation, by virtue of the equation itself, has to be dependent on the forms of the others, it is obvious that, in general, one cannot assume any of these functions to be given. Thus, for example, the above equation could not be satisfied if $f(x)$ had any other form than that which was found." (Translated from French; a misprint corrected.)

After initial results by Dhombres (unpublished), M. Sablik (1989) first determined all continuous solutions of (18) on $\mathbf{R}^{2}$ that is, if (18) is supposed to hold for all real $x, y$. It is not quite clear why Abel singled out this equation. He starts, however, from the solutions

$$
\begin{array}{lll}
f(x)=\frac{x}{2}, & \psi(x)=\phi(x)=\log x, \\
f(x)=\sqrt{1-x^{2}}, & \psi(x)=\phi(x)=\arcsin x .
\end{array}
$$

These are solutions only on subsets of the real plane (the first on $\mathbf{R}_{+}^{2}=$ $\{(x, y) \mid x>0, y>0\}$, the second on $\{(x, y) \mid x, y \in[-1,1]\}$ (there are also problems with the functions being multivalued in the second solution). So it is preferable to find the general (at least continuous) solutions of (18) on such subsets (say, on regions). Actually, Sablik (1989) has also determined all continuous solutions of (18) restricted to cartesian squares of certain intervals. 
Instead of the longish list of continuous solutions, we give here some basic ideas of the argument in the case where 0 is in the fundamental interval. The case $f(0)=0$ is uninteresting (it leads to continuous solutions $f$ which are arbitrary for $x \neq 0$ and to $\phi, \psi$ which are constant where it counts). If $f(0) \neq 0$, then (18) can be reduced, with $g(x)=f(x) / f(0)$, $\Psi(x)=\psi(f(0) x)-\psi(0)$, to

$$
\Psi(x)+\Psi(y)=\Psi[x g(y)+y g(x)]
$$

and this to

$$
g[x g(y)+y g(x)]=g(x) g(y)+c x y .
$$

The proof concludes with the determination of all continuous solutions of this equation on the considered intervals. Sablik noticed also the interesting facts that (19) is essentially the associativity equation (10) for $F(x, y)=x g(y)+y g(x)$ and that, if supposed for all $(x, y) \in \mathbf{R}^{2}$ and if $c>0$, (19) is also equivalent to the "Baxter equation"

$$
h[x h(y)+y h(x)-x y]=h(x) h(y) .
$$

All continuous solutions of (20) were determined on $\mathbf{R}^{2}$ by Volkmann and Weigel (1984) (for solutions without continuity assumptions see e.g. Benz (1987)). For linear operators on Banach algebras, (20) defines the Baxter (or 'summation') operators, connected to queuing theory and extensively researched in the last three decades (see e.g. Kingman (1966) Miller (1969)).

It would be desirable to find the continuous solutions of (18) on more general sets and also the solutions under regularity conditions weaker than continuity (say, integrability or measurability).

2. An unfinished manuscript (apparently notes to his planned book on elliptic functions) of Abel (1828) reduces the system of functional equations

$$
\begin{gathered}
\phi(x+y) \phi(x-y)=\phi(x)^{2} f(y)^{2}-\phi(y)^{2} f(x)^{2}, \\
f(x+y) f(x-y)=f(x)^{2} f(y)^{2}-c^{2} \phi(x)^{2} \phi(y)^{2}
\end{gathered}
$$

to differential equations of up to fourth order, of course under supposition of differentiability (of fourth order), that is, since the functions are complex, of analyticity. Haruki (1965) has found, also by reduction to differential equations of at most fourth order, the general (in fourth order) differentiable solutions of (21) alone.

The general continuous solution either of (21) alone or of the system (21), (22) for all $x, y \in \mathbf{C}$ is not known (to me); even less are those under regularity conditions weaker than continuity or on subsets of $\mathbf{C}^{2}$.

5. Conclusion. Summarizing, in particular in view of Sablik's (1989) recent result (see 4.1), the second part of Hilbert's Fifth Problem is largely solved. (We can say this with so much more justification, since Hilbert had put particular emphasis on eliminating the differentiability conditions from the results in Abel (1823), (1826A) and (1827).) Moreover, it gave rise to several interesting and worthwhile problems, some also solved, others (yet) unsolved. 


\section{REFERENCES}

N. H. Abel (1823), Méthode générale pour trouver des fonctions d'une seule quantité variable lorsqu'une propriété des fonctions est exprimée par une équation entre deux variables, (Norwegian). Mag. Naturvidenskab. 1 (1823), No. 1, 1-10 (Oeuvres complètes, tome I. Grøndahl \& Søn, Christiania, 1881, pp. 1-10).

(1824), Détermination d'une fonction au moyen d'une équation qui ne contient qu'une seule variable, Manuscript, Christiania, c. 1824. (Oeuvres complètes, tome II. Grøndahl \& Søn, Christiania, 1881, pp. 36-39).

(1826A), Untersuchung der Functionen zweier unabhängigen veränderlichen Grössen $x$ and $y$, wie $f(x, y)$, welche die Eigenschaft haben, da $\beta f z, f(x, y)]$ eine symmetrische Function von $x, y$ und $z$ ist, J. Reine Angew. Math. 1 (1826), 11-15. (Oeuvres complètes, tome I. Grøndahl \& Søn, Christiania, 1881, pp. 61-65).

(1826B), Unterschungen über die Reihe $1+\frac{m}{1} x+\frac{m(m-1)}{1.2} x^{2}+\frac{m(m-1)(m-2)}{1.2 .3} x^{3}+\ldots$, J. Reine Angew. Math. 1 (1826), 311-339. (Oeuvres complètes, tome I. Grøndahl \& Søn, Christiania, 1881, pp. 219-250).

$(\mathbf{1 8 2 6 C})$, Note sur la fonction $\psi x=x+\frac{x^{2}}{2^{2}}+\cdots+\frac{x^{n}}{n^{2}}+\ldots$, Manuscript, Freiberg,

1826. (Oeuvres complètes, tome II. Grøndahl \& Søn, Christiania, 1881, pp. 189-193).

(1827), Über die Functionen, die der Gleichung $\phi(x)+\phi(y)=\psi(x f y+y f x)$ genug thun, J. Reine Angew. Math. 2 (1827), 386-394. (Oeuvres complètes, tome I. Grøndahl \& Søn, Christiania, 1881, pp. 389-398). (1828), Manuscript, Christiania, c. 1828. (Oeuvres complètes, tome II. Grøndahl \& Søn, Christiania, 1881, pp. 287, 318-319).

J. Aczél (1949), Sur les opérations définies pour des nombres réels, Bull. Soc. Math. France 76 (1949), 59-64.

(1961), Über die Differenzierbarkeit der integrierbaren Lösungen gewisser Funktionalgleichungen, Ann. Univ. Sci. Budapest. Eötvös Sect. Math. 3-4 (1960-1961), 5-8.

(1966), Lectures on functional equations and their applications, Academic Press, New York-London, 1966.

(1987), A short course on functional equations, based upon recent applications to the social and behavioral sciences, Reidel, Dordrecht-Boston-Lancaster-Tokyo, 1987.

J. Aczél and J. K. Chung (1982), Integrable solutions of functional equations of a general type, Studia Sci. Math. Hungar. 17 (1982), 51-67.

J. Aczél and J. Dhombres (1989), Functional equations in several variables with applications to mathematics, information theory and to the natural and social sciences, Cambridge Univ. Press, Cambridge-New York-New Rochelle-Melbourne-Sydney, 1989.

J. Aczél and A. D. Wallace (1967), A note on generalizations of transivitive systems of transformations, Colloq. Math. 17 (1967), 29-34.

J. Andrade (1900), Sur l'équation fonctionnelle de Poisson, Bull. Soc. Math. France 28 (1900), 58-63.

W. Benz (1987), Ein Beitrag zu einem Problem von Herrn Fenyö, Abh. Math. Sem. Univ. Hamburg 57 (1987), 21-25.

A. L. Cauchy (1821), Analyse algébrique, Paris, 1821. (Oeuvres, Sér. 2, tome 3. GauthierVillars, Paris, 1897).

J. K. Chung, Pl. Kannappan and C. T. $\mathrm{Ng}$ (1985), A generalization of the cosine-sine functional equation on groups, Linear Algebra Appl. 66 (1985), 259-277.

Z. Daróczy and H. Kiesewetter (1973), Eine Funktionalgleichung von Abel und die Grundgleichung der Information, Period. Math. Hungar. 4 (1973), 25-28.

L. Fuchs (1963), Partially ordered algebraic systems, Pergamon Press, Oxford-LondonNew York-Paris, 1963.

H. Haruki (1965), Studies on certain functional equations from the standpoint of analytic function theory, Sci. Rep. Osaka Univ. 14 (1965), 1-40.

D. Hilbert (1902), Mathematical problems, Lecture delivered before the International Congress of Mathematicians at Paris in 1900, Bull. Amer. Math. Soc. 8 (1902), 437-479.

A. Járai (1986), On regular solutions of functional equations, Aequationes Math. 30 (1986), 21-54. 
M. Kac (1937), Une remarque sur les équations fonctionnelles, Comment. Math. Helv. 9 (1937), 170-171.

Pl. Kannappan (1977, 1978), On Shannon's entropy and a functional equation of Abel, Period. Math. Hungar. 8 (1977), 41-44; Addendum, Period. Math. Hungar. 9 (1978), 297298.

H. Kiesewetter (1957), Eine Bemerkung über partielle Differentiationen bei N. H. Abel, Publ. Math. Debrecen 5 (1957), 265-268.

(1961), Struktur linearer Funktionalgleichungen in Zusammenhang mit dem Abelschen Theorem, J. Reine Angew. Math. 206 (1961), 113-171.

_ (1965), Über die arc tan - Funktionalgleichung, ihre mehrdeutigen, stetigen Lösungen und eine nichtstetige Gruppe. Wiss. Z. Friedrich-Schiller-Univ. Jena Math.-Natur. Reihe 14 (1965), 417-421.

J. F. C. Kingman (1966), On the algebra of queues, J. Appl. Prob. 3 (1966), 285-326.

M. Kuczma (1968), Functional equations in a single variable, Polish Scientific Publishers, Warszawa, 1968.

M. Kuczma, B. Choczewski and R. Ger (1989), Iterative functional equations, Cambridge Univ. Press, Cambridge-New York-New Rochelle-Melbourne-Sydney, 1989.

K. Lajkó (1987), Remark on the Hosszú functional equation, Wyż. Szkoła Ped. Krakow, Rocznik Nauk-Dydakt. Prace Mat. 12 (1987), 192-193.

P. M. Lee (1964), On the axioms of information theory, Ann. Math. Statist. 35 (1964), 415-418.

L. Losonczi (1985), Remark 32: The general solution of the arc tan equation, Proc. Twenty-third Internat. Sympos. on Functional Equations (Gargnano, Italy, June 2-11, 1985). Univ of Waterloo, Centre for Information Theory, Waterloo, Ont., 1985, pp. 74-76.

J. B. Miller (1969), Baxter operators and endomorphisms on Banach algebras, J. Math. Anal. Appl. 25 (1969), 503-520.

Zs. Páles and R. W. Craigen (1989), The associativity equation revisited, Aequationes Math. (to appear).

F. Radó and J. A. Baker, (1987), Pexider's equation and aggregation of allocations, Aequationes Math. 32 (1987), 227-239.

M. Sablik (1989), The continuous solutions of a functional equation of Abel, (Contribution to Hilbert's Fifth Problem), Aequationes Math. (submitted).

P. Volkmann and H. Weigel (1984), Über ein Problem von Fenyö, Aequationes Math. 27 (1984), 135-149.

M. C. Zdun (1977), On integrable solutions of Abel's functional equation, Glas. Mat. Ser. III 12(32) (1977), 49-59.

Department of Pure Mathematics, University of Waterloo, Waterloo, Ontario, Canada N2L 3G1 
